\title{
Original
}

\section{Periodontal Regeneration Using Cultured Coral Scaffolds in Class II Furcation Defects in Dogs}

\author{
Yoshifumi Matuda $^{1)}$, Tomoharu Okamura ${ }^{2)}$, Hazime Tabata ${ }^{3)}$, Kenichiro Yasui $^{4)}$, Masayasu Tatsumura ${ }^{4)}$, Nobuhiro Kobayashi ${ }^{5)}$, \\ Tetsunari Nishikawa ${ }^{6}$ and Yoshiya Hashimoto ${ }^{7}$
}

1) Department of Oral Anatomy, Osaka Dental University, Osaka, Japan

2) Department of Oral Pathology, Osaka Dental University, Osaka, Japan

3) Department of Periodontology, Osaka Dental University, Osaka, Japan

4) Department of Orthodontics, Osaka Dental University, Osaka, Japan

${ }^{5)}$ Department of Implantology, Osaka Dental University, Osaka, Japan

6) Department of Innovation in Dental Education, Osaka Dental University, Osaka, Japan

7) Department of Biomaterials, Osaka Dental University, Osaka, Japan

(Accepted for publication, July 23, 2019)

\begin{abstract}
Autogenous bone grafting involves a second surgery and is limited by the availability of collectable bone. Coral products commercially available as bone grafts are composed of corals in their natural form. Cultured corals are recommended as a substitute to overcome the threat of extinction of natural corals. The purpose of this study was to investigate the potential of cultured coral scaffolds for periodontal tissue regeneration in class II furcation defects in dogs. The cultured coral used for this study was characterized using scanning electron microscopy (SEM), powder X-ray diffraction (XRD) and Fourier-transform infrared (FTIR) spectroscopy. Twelve mandibular premolar teeth from two female beagle dogs were used. Furcation defects of three left mandibular premolar teeth of each dog received the cultured coral. A control group consisting of the right mandibular premolar teeth from each dog received no implant material within the furcation defects. The dogs were then sacrificed 8 weeks post-surgery, and healing was evaluated histologically and radiologically using micro-computed tomography analysis. SEM micrographs showed a tri-dimensional ceramic structure with pore size ranging 50-250 mm. The surface of the cultured coral particles was covered with numerous rough, porous processes. XRD and FTIR results showed that the cultured coral exhibited both aragonite and calcite phases, referred to as dimorphism. The cultured coral particles implanted into the defects were completely resorbed and replaced by bone with either a Haversian structure or without any inflammatory reaction. A well-organized periodontal ligament bridging the new bone and cementum was regenerated to the top of the furcation space. Histopathological evaluation suggests that cultured coral scaffolds have the potential to regenerate periodontal tissue in class II furcation defects in dogs.
\end{abstract}

Key words: Bone grafts, Cultured coral scaffold, Periodontal regeneration

\section{Introduction}

Severe periodontitis is characterized by tissue resorption of both alveolar bone and cementum, which subsequently causes tooth $\operatorname{loss}^{1)}$. In these cases, spontaneous regeneration does not usually occur; thus, the management of periodontitis is based on conservative approaches such as plaque control and anti-inflammatory procedures ${ }^{1)}$. However, over the last few decades, tissue regeneration and reconstruction therapies have gathered significant attention ${ }^{2}$.

Autogenous bone grafting is considered the gold standard for the treatment of alveolar bone resorption; however, it has several disadvantages. These include the requirement of a second surgery at the donor site and as a result, bone supply is limited ${ }^{3,4}$. These problems can be circumvented by implanting artificial scaffold material at the bone defect site, which promotes osteogenesis both in quantity and quality ${ }^{5)}$. Scaffolds with porous structures are popular, as this is a key component for cell adhesion, proliferation and differentiation within tissue engi-

Correspondence to: Dr. Yoshiya Hashimoto, Department of Biomaterials, Osaka Dental University, 8-1 Kuzuhahanazono-cho Hirakata, Osaka, 573-1121, Japan; Tel: +81-72-643056 ext. 5381; Fax: +81-72-643156; E-mail: yoshiya@cc.osaka-dent.ac.jp neering $^{6,7)}$. Additionally, the presence of a rough surface on the substrate material significantly increases its propensity for osseointegration ${ }^{8,9)}$. Coral possesses many of these beneficial properties. It is composed of calcium carbonate in the form of aragonite ${ }^{10)}$ and has a rough surface at the nano level, which is advantageous for cell adhesion ${ }^{11)}$. Coral products are globally used for bone reconstruction, and Corals have shown great potential, thanks to their chemical and structural characteristics similar to that of human cancellous bone, but the clinical data presented to date is ambiguous with the positive and negative results reported ${ }^{12)}$. In our previous study ${ }^{13)}$ we analyzed the internal skeletal structure of cultured coral using micro computed tomography (micro-CT) and observed that it had tubular cavities ranging from $100-250 \mathrm{~mm}$ in length penetrating outwards. Matsuda et al. ${ }^{14)}$ used human periodontal ligament cells and demonstrated that cultured coral provided a biocompatible scaffold. However, there is little information regarding the performance of cultured coral as a scaffold for the cells of the periodontium in periodontal tissue engineering applications.

Currently, the coral products commercially available as bone grafts are composed of corals in their natural form. Coral reefs are exposed to catastrophic situations. According to research by the International Union 

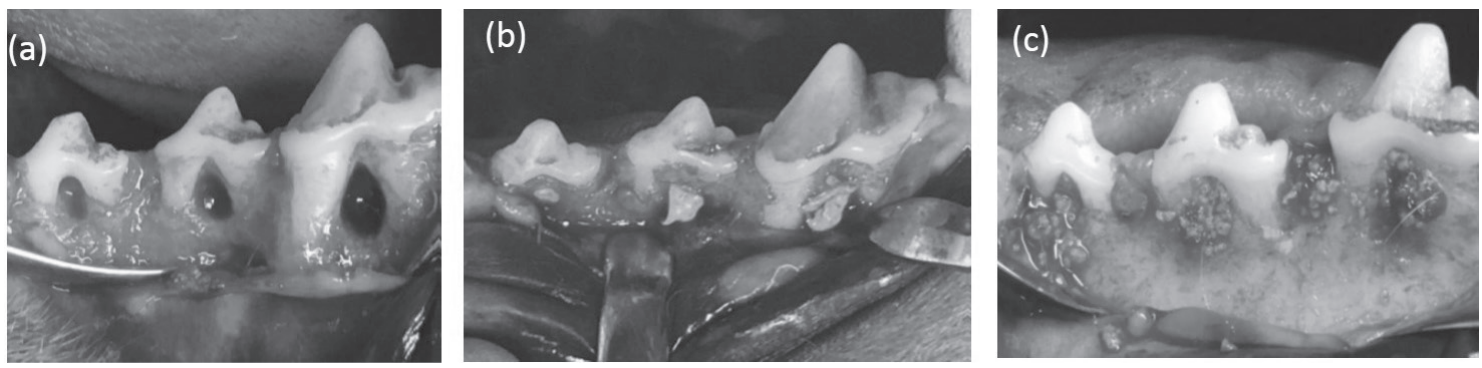

Figure 1. Photographs of the surgical procedure. (a) Preparation of the class II furcation defect (b) Placement of silicone impression material (c) Implantation of cultured coral.

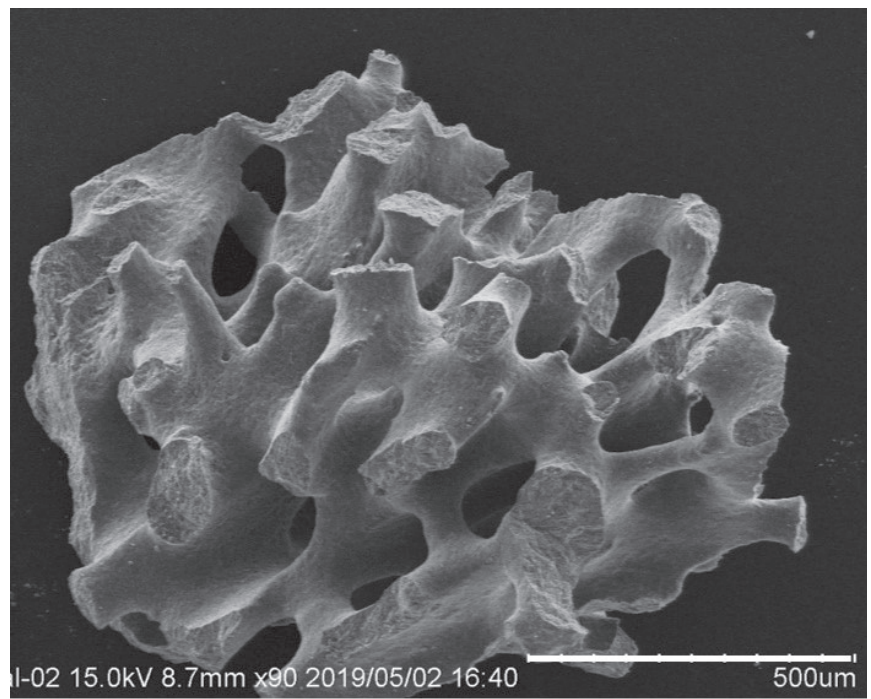

Figure 2. Scanning electron micrographs of the cultured coral.

for Conservation of Nature (IUCN) ${ }^{15}$, one third of the world's coral species are said to be at increased risk of extinction. Thus there is a dire need to look for alternatives, and cultured coral can be a suitable option. The class II furcation defects model in dogs have been used to evaluate wound healing of periodontal tissue ${ }^{16,17)}$. The purpose of this study is to investigate the potential of cultured coral scaffolds for periodontal tissue engineering in class II furcation defects in dogs.

\section{Materials}

\section{Materials and Methods}

Cultured coral (Montipora digitata) was kindly provided by the University of the Ryukyu. The coral was pulverized with a research-grade pulverizer (MN4301, Retsch, Germany). The particles (500-1,100 mm) were immersed in $1 \mathrm{~N} \mathrm{NaOH}$ to remove protein. Deproteinized cultured coral particles were washed and neutralized with phosphate-buffered saline (PBS), and further sterilized in an autoclave $\left(121^{\circ} \mathrm{C}\right.$ for $\left.20 \mathrm{~min}\right)$.

\section{Characterization of cultured coral}

Cultured coral particles were analyzed for particle size, pore distribution, and outer surface conditions. After being coated with platinum-palladium, coral samples were imaged with a scanning electron microscope (S-2700, Hitachi Ltd., Tokyo, Japan). Cultured coral particles were also characterized by the powder X-ray diffraction system (XRD-6100, Shimadzu Corp., Kyoto, Japan) under the following conditions: $40.0 \mathrm{kV}, 30.0 \mathrm{~mA}$, scan rate 2 degree/min, step size 0.05 degrees, and 3-80 degrees. Crystal phase was defined using the database from the International Centre for Diffraction Data. Fourier-transform infrared spectra (FTIR) were obtained using an FTIR spectrometer (IRAffinity-1S, Shimazu Corp., Kyoto, Japan) equipped with a diffuse reflectance unit at a resolution of $4 \mathrm{~cm}^{-1}$ with 16 scans.

\section{Surgery and implantation}

This study was performed in accordance with the guidelines for care and use of laboratory animals of the Osaka Dental University (approval no.17-05003). Twelve mandibular premolar teeth of two beagle female dogs (aged two years) were used. The surgical procedures were performed under anesthesia by intramuscular injection of medetomidine hydrochloride ( $0.5 \mathrm{mg} / \mathrm{kg}$, Domitor, Meiji Seika Co.Ltd., Tokyo, Japan) and midazolam $(0.5 \mathrm{mg} / \mathrm{kg}$, Sandoz, Yamagata, Japan $)$ as well as a local infiltration (2\% with 1:80,000 epinephrine, Aspen Japan, Tokyo, Japan). Class II furcation defects with a vertical height of $5 \mathrm{~mm}$ and a horizontal depth of $3 \mathrm{~mm}$ were created on the premolars, and scaling and root planing were performed on the exposed root surfaces (Fig. 1(a)). Next, silicone impression material was used to cover the defects and the assembly was allowed to rest for two weeks (Fig. 1(b)). Then, the cultured coral particles were embedded in the class II furcation defects of three left mandibular premolar teeth after exfoliation of the gingiva at the same site (Fig. 1(c)). The control group contained the four unembedded right mandibular premolar teeth. The fluorescent dyes calcein and alizarin were injected intraperitoneally three and seven days respectively, before the sacrifice. The bony structures were observed as a green fluorescence with a $535 \mathrm{~nm}$ filter for calcein, while the associated calcification was observed as a red fluorescence with a $590 \mathrm{~nm}$ filter for alizarin red.

\section{D microradiography and histological analysis}

Animals were euthanized 8 weeks after the surgery, and radiological evaluation was performed by micro-computed tomography analysis (SMX-130 CT, Shimadzu Corp., Kyoto, Japan). Blocks of bone specimens were mounted on the turntable. The exposure parameters were 51 $\mathrm{kV}$ and $120 \mathrm{~mA}$. Data obtained from each slice were stored at a resolution of $512 \times 512$ pixels. The generation of a $3 \mathrm{D}$ reconstruction was achieved using a volume-rendering method for morphological assessment. (TRI/3D BON software version 7, Ratoc Co.Ltd., Tokyo, Japan). Tissue blocks including teeth, bone and soft tissues were resected. Blocks were fixed in $10 \%$ buffered formalin, decalcified in $10 \%$ EDTA solution, trimmed, dehydrated, and embedded in paraffin. Serial sections of 5-7 mm were prepared in the mesio-distal plane. Sections were visualized under a fluorescence microscope (BZ9000, Keyence Corp., Tokyo, Japan) and the behavior of calcium in the bone tissue was observed by confocal laser scanning microscopy (LSM-GB200, Olympus Corp., Tokyo), followed by image processing via a computer (Vectra 386/25, Hewlett Packard, USA) to obtain tomographic images taken at levels of increasing depth of either 2 or $5 \mathrm{~mm}$ from the section surface. 


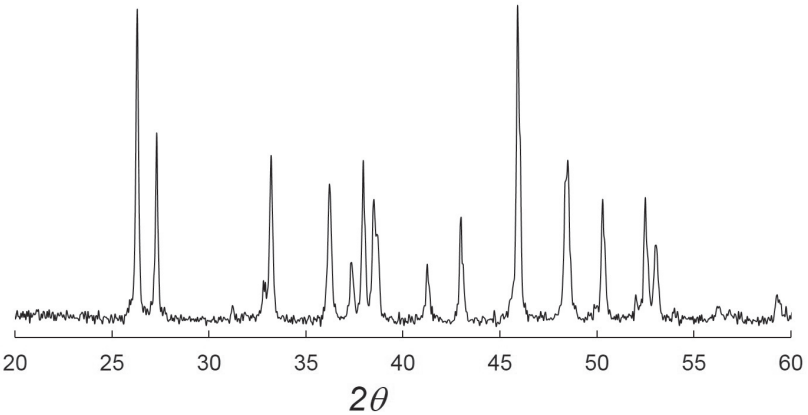

Figure 3. X-ray diffraction patterns of the cultured coral.

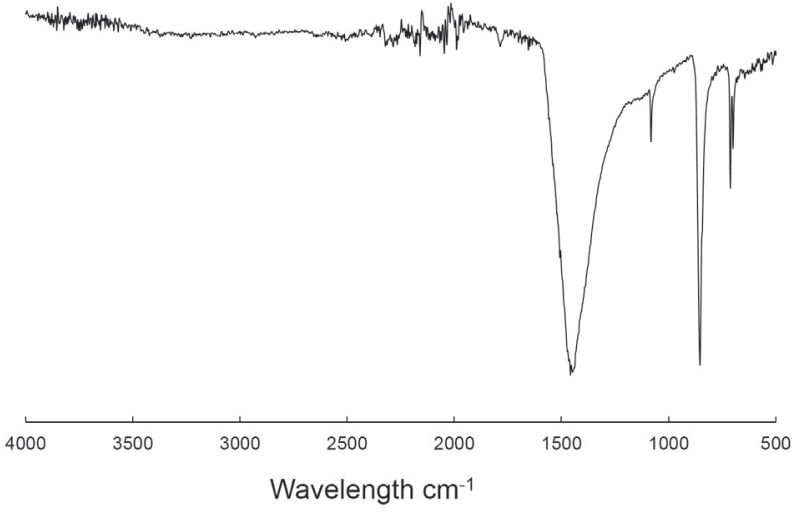

Figure 4. Fourier transform-infrared spectra of the cultured coral.
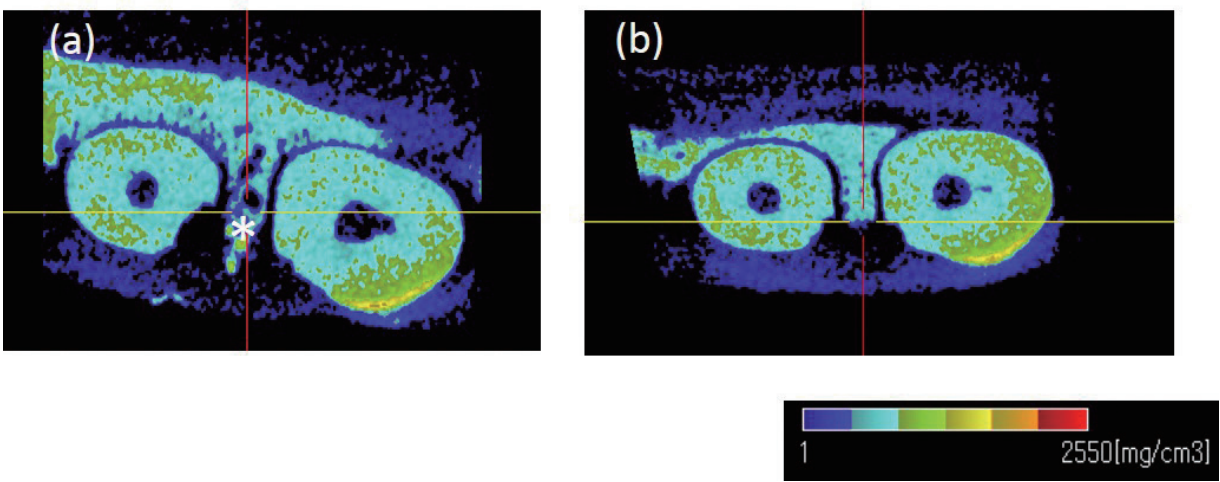

Figure 5. Micro-computed tomography cross-sectional images of three-dimensional pseudo-color representations of the mineral density. (a) The cultured coral scaffold group in cross section (b) Control group in cross section. Asterisk denotes new bone.
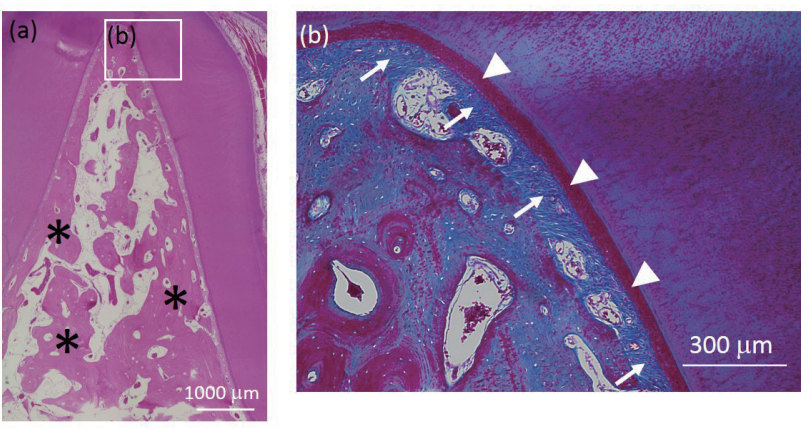

Figure 6. Photographs from the cultured coral scaffold group with (a) Hematoxylin and eosin-stain and (b) Azan stain. Asterisks, white triangles and white arrows denote the new bone, new cementum, and periodontal ligament respectively.

\section{Results}

\section{Characteristics of cultured coral}

The cultured coral particles had many pores with a diameter of 100$250 \mathrm{~mm}$ which formed an interconnected network. The surface of the cultured coral particles was covered with numerous rough, porous processes (Fig. 2). X-ray diffraction analyses of cultured coral particles revealed showed characteristic peaks with high intensity between $2 \theta$ of $20^{\circ}$ and $60^{\circ}$. All the diffraction spectra of the cultured coral conform with aragonite polymorph of calcium carbonate (Fig. 3). The FTIR spectrum of pure coral shows sharp intense peaks at $1,456 \mathrm{~cm}^{-1}$ and 854 $\mathrm{cm}^{-1}$, which may be attributed to the presence of the $\mathrm{CO}_{3}{ }^{2-}$ group. The broad band around $3,400 \mathrm{~cm}^{-1}$ and the peak around $630 \mathrm{~cm}^{-1}$ is due to the $\mathrm{OH}$ group (Fig. 4).

\section{D microradiography and histological analysis}

Quantitative imageology analysis of newly grown bone was carried out by in vivo 3D microradiography using a bone mineral density (BMD) analysis system. Filled defects in the cultured coral and control groups at eight weeks were examined by microradiography (Fig. 5(a), (b)). Warmer colors, such as red and yellow, denote high mineral density, while cooler colors such as blue and water blue indicate low mineral density. The newly slightly formed bone indicated low mineral density in the cultured coral group (Fig. 5(a)). In the control group, the new bone was slightly formed (Fig. 5(b)).

Histological analysis showed that most of the cultured coral particles were degraded and replaced throughout the entire bone defect with new bone possessing a Haversian structure and yellow bone marrow (Fig. 6(a)); tissue specimens stained with Azan illustrated that the dense fibers of connective tissue were arranged regularly and attached the new bone to the newly formed cementum (Fig. 6(b)). No signs of giant cells, infection, or other adverse tissue reactions were visible (Fig. 6(a)). Empty defects within the control group healed with a significant amount of loose connective tissue, which was gradually substituted with bone. Some empty space remained at 8 weeks (Fig. 7(a), (b)).

Both green, signifying calcein, and red, signifying alizarin red, bone lamellae were observed in the cultured coral group. Thus, the defects treated with cultured coral were filled, and calcein-labeled, newly formed bone was observed along the space of the coral (Fig. 8(a)). These findings were not observed in the control group (Fig. 8(b)). 

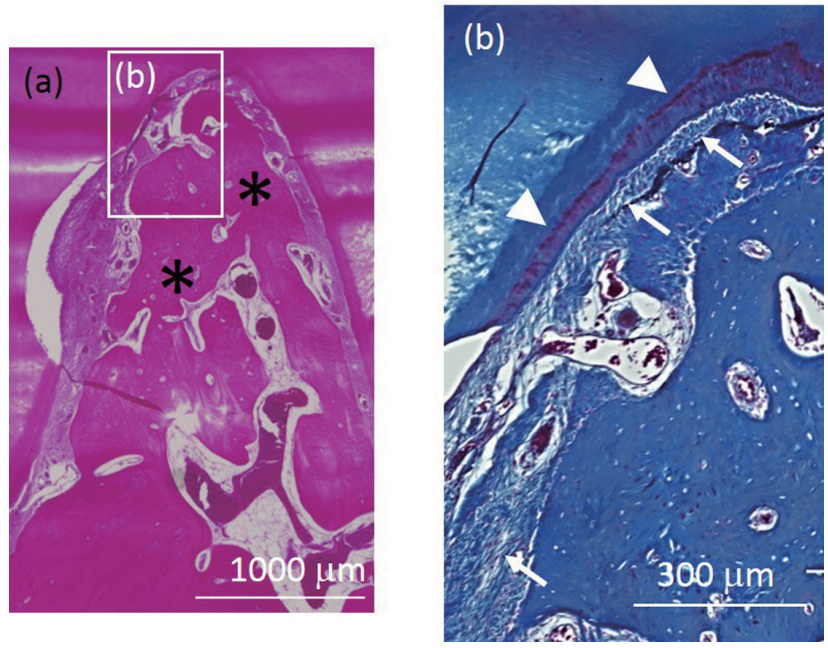

Figure 7. Photographs from control group with (a) Hematoxylin and eosin-stain and (b) Azan stain

Asterisks, white triangles and white arrows denote the new bone, new cementum, and periodontal ligament respectively.

\section{Discussion}

Periodontics generally revolves around the debridement of periodontal pockets and has traditionally not included the use of artificial scaffold material to treat patients. However, the last thirty years have seen the development of materials used in the regeneration of periodontal tissues following periodontal disease. Coral, made of calcium carbonate in the form of aragonite, has high porosity and uniform pore size with mechanical strength; leading to its considerable success in bone graft applications $^{18,19)}$. In the present study, the implanted cultured coral enhanced bone formation up to the fornix and formed periodontal tissue in complex periodontal bone defects.

Biocoral $^{\circledR}$ is a coral-derived bone graft made of natural coral skeletons from the genus Acropora of the group Madrepora. The porosity and the $3 \mathrm{D}$ structure of coral implants promotes bony ingrowth ${ }^{20)}$. Our previous study ${ }^{13)}$ showed that the internal space of the tubular cavities of cultured coral was larger than that of natural coral such as Biocoral ${ }^{\circledR}$; thus, the pore size of cultured coral particles also will be larger than that of natural coral. Preferable scaffold material for bone regeneration has a recommended pore size of $200 \mathrm{~mm}$, which support osteoblast proliferation $^{21)}$; while a pore size of over $100 \mathrm{~mm}$ was previously reported as a minimum requirement for fibrovascular regeneration ${ }^{22)}$. The role of osteogenic factors such as transforming growth factor (TGF) ${ }^{23)}$, bone morphogenic protein (BMP $)^{24)}$, and platelet rich plasma (PRP $)^{25)}$ has been described extensively in the literature. Recently, scaffold materials that function as carriers for the sustained release of osteogenic factors have also been developed ${ }^{26,27)}$. The porous structure of corals maintains the concentration of osteogenic factors at the desired location and reduces the effect of those factors on other tissues. Thus, the skeletal shape of cultured coral appears to have an internal structure appropriate for bone tissue ingrowth.

XRD studies on coral structures have revealed major peaks of calcium carbonate aragonite crystalline phase $\left(\mathrm{CaCO}_{3}\right)$, while peaks associated with the calcite phase were not observed ${ }^{28,29)}$. Studies of coral structures using FTIR spectroscopy have also shown strong absorption bands associated with calcium carbonate aragonite. The stable phase of calcium carbonate at atmospheric pressure is called calcite, having a R3c structure, while the stable phase at high pressure is called aragonite,
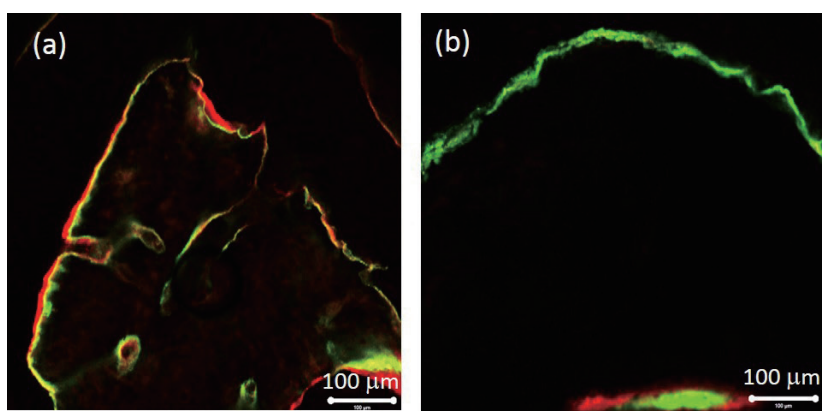

Figure 8. CLSM image of bone formation. (a) The cultured coral group (b) Control group.

having a Pmcn structure ${ }^{30)}$. Biocoral ${ }^{\circledR}$ is made from the genus Acropora and is also found only in the aragonite phase ${ }^{30)}$. Preadipocytes cultured on marine origin coral (Porites lutea), which too is found only in the aragonite phase, differentiated into an osteogenic phenotype without added bone morphogenesis inducers ${ }^{31)}$.

In this in vivo study, the implanted cultured coral particles were completely resorbed and replaced by bone in the class II furcation defects at 8 weeks. Jarmo et al. ${ }^{32)}$ demonstrated that Biocoral ${ }^{\circledR}$ was almost completely resorbed into drill-holes in the femoral condyles of adult rabbits at as early as 6 weeks, supporting our results. In our previous study ${ }^{33)}$, soaking cultured coral particles in an acid or neutral solution increased their calcium density as compared to ceramic bone in both solutions. Therefore, coral may be considered as a good bioabsorptive material.

To summarize, the space-providing, cultured coral scaffold is resorbed within 8 weeks without any inflammatory reaction. The cultured coral scaffold provides for bone and cementum regeneration with a functionally-oriented periodontal ligament. Ankylosis, defined as the obliteration of the periodontal ligament space and progressive replacement of dentin and cementum by bone ${ }^{34)}$, was not observed in this study. The histopathological evaluation suggests that the cultured coral scaffold has a periodontal regeneration potential as demonstrated in class II mandibular furcation defects. Further studies with short-term observations are required to clarify the effects of the cultured coral scaffold in class II furcation defects.

\section{Acknowledgements}

This study was supported by MEXT/JSPS KAKENHI Grant number 16K11667 and 18K17092.

\section{Conflict of Interest}

The authors have declared that no COI exists.

\section{References}

1. Chapple ILC, Van Der Weijden F, Doerfer C, Herrera D, Shapira L, Polak D, Madianos P, Louropoulou A, Machtei E, Donos N, Greenwell H, Van Winkelhoff AJ, Eren Kuru B, Arweiler N, Teughels W, Aimetti M, Molina A, Montero E and Graziani F. Primary prevention of periodontitis: Managing gingivitis. J Clin Periodontol 42: S71-S76, 2015

2. Kornman KS, Giannobile WV and Duff GW. Quo vadis: what is the future of periodontics? How will we get there? Periodontol 2000 75: 353-371, 2017

3. Sakai K, Hashimoto Y, Baba S, Nishiura A and Matsumoto N. Effects on bone regeneration when collagen model polypeptides are 
combined with various sizes of alpha-tricalcium phosphate particles. Dent Mater J 30: 913-922, 2011

4. Weiland AJ, Phillips TW and Randolph MA. Bone grafts: A radiologic, histologic, and biomechanical model comparing autografts, allografts, and free vascularized bone grafts. Plast Reconstr Surg 74: 368-379, 1984

5. Nishikawa T, Kokubu M, Kato H, Imai $\mathrm{K}$ and Tanaka A. Confocal laser scanning microscopy in study of bone calcification. Appl Surf Sci 262: 64-68, 2012

6. Annabi N, Nichol JW, Zhong X, Ji C, Koshy S, Khademhosseini A and Dehghani F. Controlling the porosity and microarchitecture of hydrogels for tissue engineering. Tissue Eng - Part B: Rev 16: 371383,2010

7. Hutmacher DW. Scaffolds in tissue engineering bone and cartilage. Biomaterials 21: 2529-2543, 2000

8. Albrektsson T, Brånemark PI, Hansson HA and Lindström J. Osseointegrated titanium implants: Requirements for ensuring a long-lasting, direct bone-to-implant anchorage in man. Acta Orthop Scand 52: 155-170, 1981

9. Wirth C, Grosgogeat B, Lagneau C, Jaffrezic-Renault N and Ponsonnet $\mathrm{L}$. Biomaterial surface properties modulate in vitro rat calvaria osteoblasts response: Roughness and or chemistry?. Mater Sci Eng C 28: 990-1001, 2008

10. Julia V, Maharani DA, Kartasasmita RE and Latief BS. The use of coral scaffold in oral and maxillofacial surgery: A review. J Int Dent Med Res 9: 427-435, 2016

11. Ono T, Nishikawa T, Tanaka A and Matsumoto N. Histological reaction to porous coral and ceramic bone. J Oral Tissue Engin 11: 8597, 2013

12. Pountos I and Giannoudis PV. Is there a role of coral bone substitutes in bone repair?. Injury 47: 2606-2613, 2016

13. Nishikawa T, Okamura T, Masuno K, Matsumoto H, Hirose M, Uemura N, Yasuda N, Hidaka M, Baba S, Imai K and Tanaka A. Comparative study of physical and morphological characteristics of cultured and natural coral as a bone augmentation scaffold. J Oral Tissue Engin 14: 107-113, 2016

14. Matsuda Y, Nishikawa T, Okamura T, Tominaga K, Wato M, Tabata H, Umeda M, Okusa N, Imai K, Tanaka A and Tamura I. Comparative study of tissue affinity, chemical characteristics of cultured and natural coral as a bioabsorbable scaffold. J Oral Tissue Engin 14: 164-170, 2017

15. David O and Gabriel G. Resilience assessment of coral reefs. Rapid assessment protocol for coral reefs, focusing on coral bleaching and thermal stress. IUCN Resilience Science Group Working Paper Series - No 5, 2009

16. Bogle G, Garrett S, Stoller NH, Swanbom DD, Fulfs JC, Rodgers PW, Whitman S, Dunn RL, Southard GL and Polson AM. Periodontal regeneration in naturally occurring class II furcation defects in beagle dogs after guided tissue regeneration with bioabsorbable barriers. J Periodontol 68: 536-544, 1997

17. Kosen Y, Miyaji H, Kato A, Sugaya T and Kawanami M. Application of collagen hydrogel/sponge scaffold facilitates periodontal wound healing in class II furcation defects in beagle dogs. J Periodontal Res 47: 626-634, 2012

18. Fu K, Xu Q, Czernuszka J, Triffitt JT and Xia Z. Characterization of a biodegradable coralline hydroxyapatite/calcium carbonate composite and its clinical implementation. Biomed Mater 8, 2013: doi: 10.1088/1748-6041/8/6/065007

19. Zhu H, Schulz J and Schliephake H. Human bone marrow stroma stem cell distribution in calcium carbonate scaffolds using two different seeding methods. Clin Oral Implants Res 21: 182-188, 2010

20. Guillemin G, Meunier A, Dallant P, Christel P, Pouliquen JC and Sedel L. Comparison of coral resorption and bone apposition with two natural corals of different porosities. J Biomed Mater Res 23: 765-779, 1989

21. Ishaug SL, Crane GM, Miller MJ, Yasko AW, Yaszemski MJ and Mikos AG. Bone formation by three-dimensional stromal osteoblast culture in biodegradable polymer scaffolds. J Biomed Mater Res A 36: 17-28, 1997

22. Hulbert S, Young F, Mathews R, Klawitter J, Talbert C and Stelling F. Potential of ceramic materials as permanently implantable skeletal prostheses. J Biomed Mater Res 4: 433-456, 1970

23. Lalani Z, Wong M, Brey EM, Mikos AG and Duke P. Spatial and temporal localization of transforming growth factor- $\beta 1$, bone morphogenetic protein-2, and platelet-derived growth factor-A in healing tooth extraction sockets in a rabbit model. J Oral Maxillofac Surg 61: 1061-1072, 2003

24. Yamachika E, Tsujigiwa H, Shirasu N, Ueno T, Sakata Y, Fukunaga J, Mizukawa N, Yamada M and Sugahara T. Immobilized recombinant human bone morphogenetic protein-2 enhances the phosphorylation of receptor-activated Smads. J Biomed Mater Res A 88: 599-607, 2009

25. Lacci KM and Dardik A. Platelet-rich plasma: support for its use in wound healing. Yale J Biology Med 83: 1, 2010

26. Kobayashi N, Hashimoto Y, Otaka A, Yamaoka T and Morita S. Porous alpha-tricalcium phosphate with immobilized basic fibroblast growth factor enhances bone regeneration in a canine mandibular bone defect model. Materials 9, 2016: doi: 10.3390/ma9100853

27. Omata K, Matsuno T, Asano K, Hashimoto Y, Tabata Y and Satoh T. Enhanced bone regeneration by gelatin- $\beta$-tricalcium phosphate composites enabling controlled release of bFGF. J Tissue Eng Regen Med 8: 604-611, 2014

28. Mansur HS, Mansur AA and Pereira M. XRD, SEM/EDX and FTIR characterization of Brazilian natural coral. Key Eng Mater 284: 4346, 2005

29. Sivakumar M, Kumar TS, Shantha K and Rao KP. Development of hydroxyapatite derived from Indian coral. Biomaterials 17: 17091714, 1996

30. Wu Y-C, Lee T-M, Chiu K-H, Shaw S-Y and Yang C-Y. A comparative study of the physical and mechanical properties of three natural corals based on the criteria for bone-tissue engineering scaffolds. J Mater Sci Mater Med 20: 1273-1280, 2009

31. Birk RZ, Abramovitch-Gottlib L, Margalit I, Aviv M, Forti E, Geresh S and Vago R. Conversion of adipogenic to osteogenic phenotype using crystalline porous biomatrices of marine origin. Tissue Eng 12: 21-31, 2006

32. Gunn JM, Rekola J, Hirvonen J and Aho AJ. Comparison of the osteoconductive properties of three particulate bone fillers in a rabbit model: Allograft, calcium carbonate $\left(\right.$ Biocoral $^{\circledR}$ ) and S53P4 bioactive glass. Acta Odontol Scand 71: 1238-1242, 2013

33. Nishikawa T, Okamura T, Masuno K, Tominaga K, Wato M, Uobe KI, Imai K, Takeda S, Kono T, Morita S, Hidaka M and Tanaka A. Tissue affinity and chemical characteristics of coral. Nano Biomed 3: 231-236, 2011

34. Kim CS, Choi SH, Cho KS, Chai JK, Wikesjö UME and Kim CK. Periodontal healing in one-wall intra-bony defects in dogs following implantation of autogenous bone or a coral-derived biomaterial. J Clin Periodontol 32: 583-589, 2005 
J.Hard Tissue Biology Vol. 28(4): 329-334, 2019 